\title{
Accreting Plasmas in Black Hole Magnetospheres
}

\author{
M. Takahashi \\ Department of Physics and Astronomy, Aichi University of Education, \\ Kariya, Aichi 448-8542, Japan
}

\begin{abstract}
We present a fully relativistic study on the standing shock formation for magnetohydrodynamical plasmas in a stationary and axisymmetric black hole magnetosphere. We express all the postshock physical quantities in terms of the relativistic compression ratio. Then, the downstream state of a shocked plasma is determined by the upstream state of the accretion. We also discuss the dragging-effects of the rotating black hole on the shock conditions.
\end{abstract}

\section{Introduction}

We study the central engine of active galactic nuclei (AGNs). There are many suggestions for the existence of supermassive black holes and accreting plasmas in the central regions. In this paper, to explain the activities of AGNs, we introduce the effect of magnetic fields and discuss the central engine as "a black hole magnetosphere", where magnetized plasma (e.g., an accretion disk and its corona) surrounds a black hole and an accretion and wind/jet would be generated from the surrounding plasma. We treat plasmas accreting onto a black hole and formulate the general relativistic magnetohydrodynamical (MHD) shock conditions. We expect that a very hot plasma area appears near the event horizon by the shock. The emission from this hot plasma would directly bring information of the black hole to us.

The formation of shocks is based on the existence of multi-magnetosonic points in the accretion solution. This is because an accretion initially ejected from a plasma source with a low velocity must be terminally superfast magnetosonic at the event horizon. At the shock front, the flow transits from supermagnetosonic to sub-magnetosonic, so the accretion with a shock must pass through a magnetosonic point on each side of the shock front.

The trans-magnetosonic MHD flow solution was discussed by Takahashi (2000). Along the magnetic field line, the five physical quantities are conserved: the total energy and angular momentum, the angular frequency of the magnetic field line, the particle number flux per magnetic flux tube, and entropy (see Camenzind 1986). When these conserved quantities are given at the plasma source, the locations of the fast/slow magnetosonic points and the Alfvén points are determined. Takahashi (2000) demonstrated multi-magnetetosonic point solutions and found two regimes of accreting flows, that is, "hydro-like" and "magneto-like" accretion solutions. The hydro-like accretion would transit to magneto-like accretion by the shock formation. 
In this paper, we apply the shock conditions for special relativistic MHD jets derived by Appl \& Camenzind (1988) to the shock conditions for general relativistic MHD accretions onto a Kerr black hole. Our main purpose of this extension is to be clear about "the general relativistic effects" in MHD shock conditions. We try to express all the postshock physical quantities in terms of the relativistic compression ratio. This compression ratio is the solution of a polynomial of seventh degree; this situation is the same as the special relativistic case.

\section{Basic Equations of Relativistic Plasma Flow}

We assume a stationary and axisymmetric magnetosphere and ignore its selfgravity. We also require infinite conductivity for the plasma flow. The background metric is written in Boyer-Lindquest coordinates. We are using geometrized units $(c=G=1)$. The basic equations of relativistic plasma flow are as follows:

(i) the particle number conservation

$$
\left(n u^{\alpha}\right)_{; \alpha}=0
$$

(ii) the conservation of total energy and momentum

$$
T_{; \beta}^{\alpha \beta}=0
$$

where the energy-momentum tensor is given by

$$
T^{\alpha \beta}=n \mu u^{\alpha} u^{\beta}-P g^{\alpha \beta}+\frac{1}{4 \pi}\left(F_{\lambda}^{\alpha} F^{\lambda \beta}+\frac{1}{4} g^{\alpha \beta} F^{2}\right),
$$

$n$ is the proper particle number density, $\mu$ is the relativistic specific enthalpy, $P$ is the pressure, and $F^{2}=F^{\mu \nu} F_{\mu \nu}$; and

(iii) the MHD condition

$$
u^{\beta} F_{\alpha \beta}=0 .
$$

The magnetic field and electric field seen in the shock rest frame are

$$
\begin{aligned}
B_{\alpha} & \equiv \frac{1}{2} \eta_{\alpha \beta \gamma \delta} k^{\beta} F^{\gamma \delta}, \quad \text { and } \\
E_{\alpha} & \equiv F_{\alpha \beta} k^{\beta},
\end{aligned}
$$

where $k^{\alpha}=(1,0,0,0)$ is the time-like Killing vector and $\eta_{\alpha \beta \gamma \delta} \equiv \sqrt{-g} \epsilon_{\alpha \beta \gamma \delta}$.

Further, we introduce the angular velocity of the magnetosphere (see Bekenstein \& Oron 1978; Camenzind 1986)

$$
\Omega_{F} \equiv-\frac{F_{t r}}{F_{\phi r}}=-\frac{F_{t \theta}}{F_{\phi \theta}}
$$

Then, we obtain the following useful expressions

$$
\begin{aligned}
& E_{\theta}=\frac{-\sqrt{-g} \Omega_{F}}{g_{t t}+g_{t \phi} \Omega_{F}} B^{r}, \quad \text { and } \\
& E_{r}=\frac{\sqrt{-g} \Omega_{F}}{g_{t t}+g_{t \phi} \Omega_{F}} B^{\theta} .
\end{aligned}
$$




\section{The Jump Conditions}

We discuss the properties of MHD shocks associated with accretion flows. The accretion is originated from the disk surface located on the equatorial plane of a black hole. We expect that the strong shock would be produced somewhere between the plasma source and the event horizon.

In a complete solution of the accretion which includes a shock, the flow must satisfy a set of conditions on either side of the discontinuity. The jump conditions for arbitrary shocks in a relativistic MHD flow are

$$
\begin{aligned}
& {\left[n u^{\alpha}\right] n_{\alpha}=0 \quad \text { - the particle number conservation, }} \\
& {\left[T^{\alpha \beta}\right] n_{\alpha}=0 \quad \text { - the energy momentum conservation, }} \\
& {[\mathrm{E}] \times \mathrm{n}=0 \text { - the continuity relations for the electric field, and }} \\
& {[B] \cdot n=0 \quad \text { - the continuity relations for the magnetic field, }}
\end{aligned}
$$

where $n^{\alpha}=\left(n_{0}, \mathrm{n}\right)$.

We consider a shock located in the $(r, \theta)$-plane in Boyer-Lindquest coordinates. We assume that the downstream flow velocity is radial (normal to the event horizon) and that the shock front is perpendicular to the downstream flow, $\mathrm{n}=(1,0,0)$. Then, we set

$$
\begin{aligned}
u_{1}^{\alpha} & =\left(u_{1}^{t}, u_{1}^{r}, u_{1}^{\theta}, u_{1}^{\phi}\right), \\
u_{2}^{\alpha} & =\left(u_{2}^{t}, u_{2}^{r}, 0, u_{2}^{\phi}\right), \\
B_{1}^{\alpha} & =\left(B^{r}, B_{1}^{\theta}, B_{1}^{\phi}\right), \\
B_{2}^{\alpha} & =\left(B^{r}, 0, B_{2}^{\phi}\right), \\
E_{1}^{\alpha} & =\left(E_{1}^{r}, E^{\theta}, 0\right), \quad \text { and } \\
E_{2}^{\alpha} & =\left(0, E^{\theta}, 0\right),
\end{aligned}
$$

where Eqs. (12) and (13) have been used. From Eq. (8), we see that $\Omega_{F}$ does not change across the shock. Equations (10) and (11) evaluated in the shock rest frame yield the following relations

$$
\begin{gathered}
n_{1} u_{1}^{r}=n_{2} u_{2}^{r} \\
n_{1} \mu_{1}\left(u^{r} u_{r}\right)_{1}-P_{1}+\frac{1}{8 \pi g_{t t}}\left(-E_{1}^{r} E_{r 1}+B_{1}^{\theta} B_{\theta 1}+B_{1}^{\phi} B_{\phi 1}\right) \\
=n_{2} \mu_{2}\left(u^{r} u_{r}\right)_{2}-P_{2}+\frac{1}{8 \pi g_{t t}}\left(B_{2}^{\phi} B_{\phi 2}\right) \\
n_{1} \mu_{1} u_{1}^{r} u_{1}^{\theta}-\frac{1}{4 \pi g_{t t}}\left(B^{r} B_{1}^{\theta}+E_{1}^{r} E^{\theta}\right)=0, \\
n_{1} \mu_{1} u_{1}^{r} u_{1}^{\phi}-\frac{1}{4 \pi g_{t t}}\left(B_{1}^{\phi} B^{r}\right)=n_{2} \mu_{2} u_{2}^{r} u_{2}^{\phi}-\frac{1}{4 \pi g_{t t}}\left(B_{2}^{\phi} B^{r}\right), \text { and } \\
n_{1} \mu_{1} u_{1}^{r} u_{1}^{t}-\frac{1}{4 \pi g_{t t}}\left(\frac{B_{\phi 1} E_{\theta}}{\sqrt{-g}}+B_{1}^{t} B^{r}\right)=n_{2} \mu_{2} u_{2}^{r} u_{2}^{t}-\frac{1}{4 \pi g_{t t}}\left(\frac{B_{\phi 2} E_{\theta}}{\sqrt{-g}}+B_{2}^{t} B^{r}\right)
\end{gathered}
$$




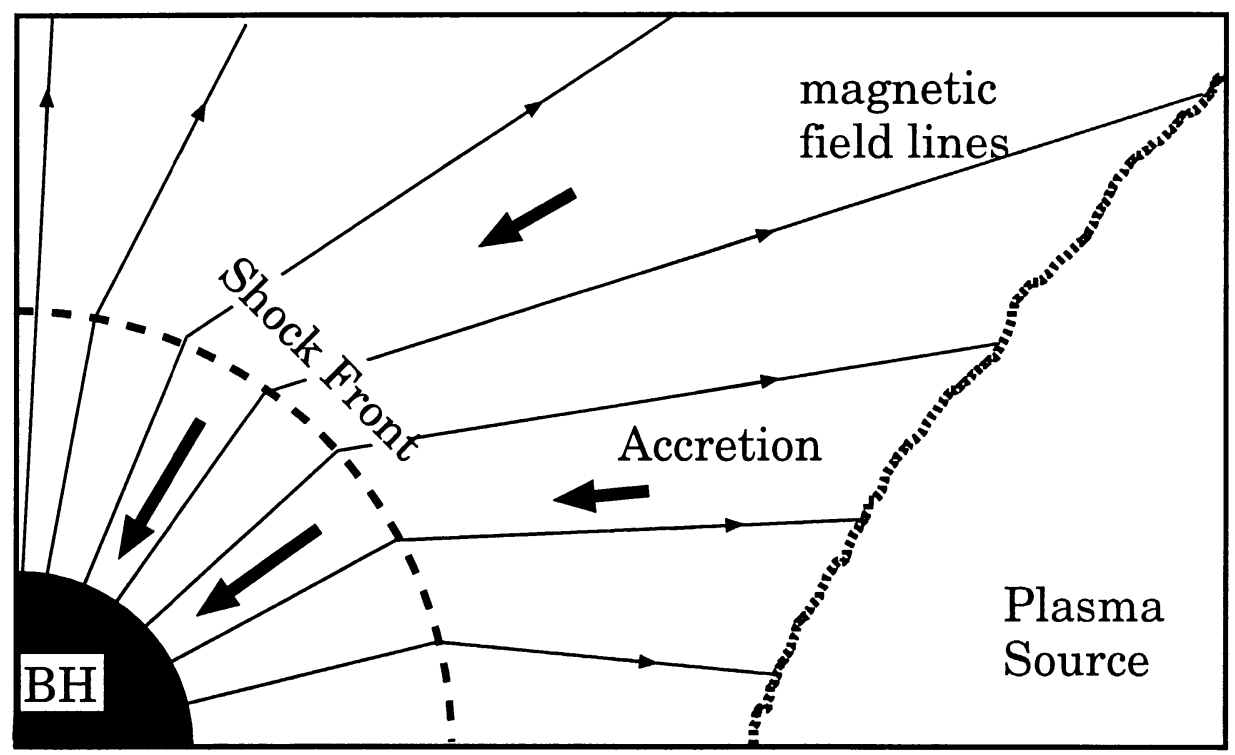

Figure 1. A schematic picture of accretion onto a black hole with a shock front.

where the subscripts " 1 " and " 2 " denote the preshock and the postshock quantities, respectively, and from the MHD conditions, we also obtain

$$
\begin{aligned}
& E_{r 1} u_{t 1}+\sqrt{-g}\left(B_{1}^{\phi} u_{1}^{\theta}-B_{1}^{\theta} u_{1}^{\phi}\right)=0, \\
& \left(B^{r} u_{1}^{\phi}-B_{1}^{\phi} u_{1}^{r}\right) \frac{1}{u_{t 1}}=\frac{-E_{\theta}}{\sqrt{-g}}=\left(B^{r} u_{2}^{\phi}-B_{2}^{\phi} u_{2}^{r}\right) \frac{1}{u_{t 2}}, \\
& \left(g_{t \phi} E_{\theta}+\sqrt{-g} B^{r}\right) u_{1}^{\theta}+\left(g_{t \phi} E_{r 1}-\sqrt{-g} B_{1}^{\theta}\right) u_{1}^{r}=0, \text { and } \\
& E_{r 1} u_{1}^{r}+E_{\theta 1} u_{1}^{\theta}=0 .
\end{aligned}
$$

\section{Dimensionless Parameters}

For any stationary and axisymmetric system we can define conserved flux vectors for energy and angular momentum about the axis of symmetry (see Blandford $\&$ Znajek 1977). From the conservation of total energy and momentum (2) and the Killing equation $\chi_{\mu ; \nu}+\chi_{\nu ; \mu}=0$, where $\chi^{\mu}$ is a Killing vector, it follows that $\left(\chi_{\mu} T^{\mu \nu}\right)_{; \nu}=0$. Thus, we define the conserved energy flux

$$
\mathcal{E}^{\mu}=T^{\mu \nu} k_{\nu}=T_{t}^{\mu}=\left(T_{t}^{\mu}\right)_{\mathrm{em}}+\left(T_{t}^{\mu}\right)_{\text {fluid }}
$$

and angular momentum flux

$$
-\mathcal{L}^{\mu}=T^{\mu \nu} m_{\nu}=T_{\phi}^{\mu}=\left(T_{\phi}^{\mu}\right)_{\mathrm{em}}+\left(T_{\phi}^{\mu}\right)_{\text {fluid }}
$$


where $m^{\nu}$ is the axial Killing vector with Boyer-Lindquist component $(0,0,0,1)$. In the case of MHD flows, these fluxes are composed of the electromagnetic part labeled by "em" and the fluid part labeled by "fluid".

The fluid and electromagnetic parts of the radial component of the energy flux $\mathcal{E}^{r}$ are

$$
\begin{aligned}
\mathcal{E}_{\text {fluid }}^{r} & \equiv\left(T_{t}^{r}\right)_{\text {fluid }}=n \mu u^{r} u_{t}, \quad \text { and } \\
\mathcal{E}_{\mathrm{em}}^{r} & \equiv\left(T_{t}^{r}\right)_{\mathrm{em}}=-\frac{B_{\phi} E_{\theta}}{4 \pi \sqrt{-g}}=\frac{B_{\phi} B^{r} \Omega_{F}}{4 \pi\left(g_{t t}+g_{t \phi} \Omega_{F}\right)},
\end{aligned}
$$

respectively, and the fluid and electromagnetic parts of the radial component of the angular momentum flux $\mathcal{L}^{r}$ are

$$
\begin{aligned}
-\mathcal{L}_{\text {fluid }}^{r} & \equiv\left(T_{\phi}^{r}\right)_{\text {fluid }}=n \mu u^{r} u_{\phi}, \quad \text { and } \\
-\mathcal{L}_{\mathrm{em}}^{r} & \equiv\left(T_{\phi}^{r}\right)_{\mathrm{em}}=-\frac{B_{\phi}}{4 \pi g_{t t}}\left(\frac{g_{t \phi} E_{\theta}}{\sqrt{-g}}+B^{r}\right)=-\frac{B_{\phi} B^{r}}{4 \pi\left(g_{t t}+g_{t \phi} \Omega_{F}\right)},
\end{aligned}
$$

respectively, where we have used the relation (8).

From the energy momentum conservation at the shock front, we have $\left(T^{r t}\right)_{1}=$ $\left(T^{r t}\right)_{2} . T^{r t}$ can be reduced as

$$
\begin{aligned}
T^{r t} & =g^{t t}\left(\mathcal{E}_{\text {fluid }}^{r}+\mathcal{E}_{\mathrm{em}}^{r}\right)-g^{t \phi}\left(\mathcal{L}_{\text {fluid }}^{r}+\mathcal{L}_{\mathrm{em}}^{r}\right) \\
& =g^{t t}\left\{n \mu u^{r} u_{t}(1-\omega \ell)+\frac{B_{\phi} B^{r}\left(\Omega_{F}-\omega\right)}{4 \pi\left(g_{t t}+g_{t \phi} \Omega_{F}\right)}\right\},
\end{aligned}
$$

where $\ell \equiv-u_{\phi} / u_{t}$ is the specific angular momentum of the plasma and $\omega \equiv$ $-g_{t \phi} / g_{\phi \phi}$ is the angular velocity of the zero angular momentum observer (ZAMO) with respect to a distant observer.

Here, we define dimensionless parameters. First, we define the "magnetization parameter", which denotes the ratio of the Poynting flux and the total mass-energy flux seen by ZAMO:

$$
\sigma \equiv \frac{\left(\mathcal{E}^{r}-\omega \mathcal{L}^{r}\right)_{\mathrm{em}}}{\left(\mathcal{E}^{r}-\omega \mathcal{L}^{r}\right)_{\text {fluid }}}
$$

Thus, we can express Eq. (33) as

$$
T^{r t}=g^{t t}\left(\mathcal{E}^{r}-\omega \mathcal{L}^{r}\right)_{\text {fluid }}(1+\sigma) .
$$

From these definitions and Eq. (8), we get

$$
\sigma=\frac{B^{r} B_{\phi}}{4 \pi n \mu u^{r} u^{t}} \mathcal{A}
$$

where

$$
\mathcal{A} \equiv \frac{-\left(g_{t \phi}+g_{\phi \phi} \Omega_{F}\right)}{\rho_{w}^{2}\left(g_{t t}+g_{t \phi} \Omega_{F}\right)}
$$


From Eqs. (26) and (8), we also get

$$
B^{r}=\frac{u^{r}}{u^{t}}\left\{\frac{g_{t t}+g_{t \phi} \Omega_{F}}{g_{t t}\left(\Omega-\Omega_{F}\right)}\right\} B^{\phi},
$$

where $\Omega \equiv u^{\phi} / u^{t}$ is the angular velocity of the fluid. Then, Eq.(37) can be reduced to

$$
\sigma=\frac{\left(B_{\phi}\right)^{2}}{4 \pi n \mu\left(u_{t}\right)^{2}}\left\{\frac{-g_{\phi \phi}\left(\Omega_{F}-\omega\right)}{\left(\rho_{w}^{2}\right)^{2}\left(\Omega_{F}-\Omega\right)}\right\} .
$$

For a local observer corotating with the magnetic field line $\left(\omega=\Omega_{F}\right)$, this parameter becomes zero.

Next, we define the following dimensionless parameters:

$$
\begin{aligned}
x & \equiv \frac{B^{r} B_{r}}{4 \pi n \mu u^{r} u_{r}}, \\
\xi & \equiv \frac{n_{2} u_{2}^{t}}{n_{1} u_{1}^{t}} \\
q & \equiv \frac{B_{2}^{\phi}}{B_{1}^{\phi}}=\frac{B_{\phi 2}}{B_{\phi 1}}, \quad \text { and } \\
\zeta & \equiv \frac{\mu_{2} u_{2}^{t}}{\mu_{1} u_{1}^{t}},
\end{aligned}
$$

where $\xi$ is the shock frame compression ratio and $q$ is the amplification factor for the transverse magnetic field.

From Eqs. (20), (24), (26), we obtain

$$
1-\zeta=\sigma_{1}(q-1)
$$

which is the same expression as Eq. (29) of Appl \& Camenzind (1988). From Eqs. (23) and (37), we obtain

$$
\begin{aligned}
\Omega_{1}-\zeta \Omega_{2} & =\frac{-B_{1}^{\phi} B^{r}}{4 \pi n_{1} \mu_{1} u_{1}^{r} u_{1}^{t} g_{t t}}(q-1) \\
& =x_{1}(q-1)\left(\frac{\Omega_{F}-\Omega_{1}}{g_{t t}+g_{t \phi} \Omega_{F}}\right),
\end{aligned}
$$

and from Eqs. (26) and (39), we also obtain

$$
\Omega_{2}-\Omega_{F}=\frac{q}{\xi}\left(\Omega_{1}-\Omega_{F}\right) .
$$

Thus, from Eqs. (47) and (48), we obtain

$$
q=\frac{\xi\left\{\mathcal{R}\left(g_{t t}+g_{t \phi} \Omega_{F}\right)-x_{1}\right\}}{\zeta\left(g_{t t}+g_{t \phi} \Omega_{F}\right)-x_{1} \xi}
$$


where $\mathcal{R} \equiv\left(\zeta \Omega_{F}-\Omega_{1}\right) /\left(\Omega_{F}-\Omega_{1}\right)$. From Eqs. (47) and (48), we also obtain

$$
\begin{aligned}
\Omega_{2} & =\frac{1}{\zeta}\left\{\Omega_{1}-x_{1}(q-1)\left(\frac{\Omega_{F}-\Omega_{1}}{g_{t t}+g_{t \phi} \Omega_{F}}\right)\right\} \\
& =\frac{\left(g_{t t}+g_{t \phi} \Omega_{F}\right) \Omega_{1}+x_{1}\left(\Omega_{F}-\Omega_{1}\right)-x_{1} \xi \Omega_{F}}{\zeta\left(g_{t t}+g_{t \phi} \Omega_{F}\right)-x_{1} \xi} .
\end{aligned}
$$

The normalization of the 4-velocity gives an equation for $u_{2}^{t}$ :

$$
u_{2}^{t}=\left\{g_{t t}+2 g_{t \phi} \Omega_{2}+g_{\phi \phi} \Omega_{2}^{2}+\frac{\left(u^{r} u_{r}\right)_{1}}{\xi^{2}\left(u_{1}^{t}\right)^{2}}\right\}^{-1 / 2} .
$$

When $\xi$ and $\zeta$ are determined, $u_{2}^{t}$ is obtained.

From Eq. (21), we obtain

$$
1-\frac{\zeta}{\xi}-\frac{P_{1}-P_{2}}{n_{1} \mu_{1}\left(u^{r} u_{r}\right)_{1}}+\frac{\left(-E^{r} E_{r}+B^{\theta} B_{\theta}+B^{\phi} B_{\phi}\right)_{1}-\left(B^{\phi} B_{\phi}\right)_{2}}{8 \pi n_{1} \mu_{1}\left(u^{r} u_{r}\right)_{1} g_{t t}}=0 .
$$

Using the relations

$$
\begin{aligned}
-E^{r} E_{r}+B^{\theta} B_{\theta} & =\frac{g_{t t} \alpha}{\left(g_{t t}+g_{t \phi} \Omega_{F}\right)^{2}} B^{\theta} B_{\theta}, \quad \text { and } \\
\left(B^{\phi} B_{\phi}\right)_{1}-\left(B^{\phi} B_{\phi}\right)_{2} & =\left(B^{\phi} B_{\phi}\right)_{1}\left(1-q^{2}\right)
\end{aligned}
$$

where $\alpha \equiv g_{t t}+2 g_{t \phi} \Omega_{F}+g_{\phi \phi} \Omega_{F}^{2}$, we get

$$
1-\frac{\zeta}{\xi}=\Pi-\mathcal{X}_{1}+\left(q^{2}-1\right) \mathcal{T}_{1}
$$

with

$$
\begin{aligned}
\Pi & \equiv \frac{P_{1}-P_{2}}{n_{1} \mu_{1}\left(u^{r} u_{r}\right)_{1}} \\
& =1-\frac{1}{\xi}+\frac{\sigma_{1}}{\xi}(q-1)+\mathcal{X}_{1}-\left(q^{2}-1\right) \mathcal{T}_{1} \\
\mathcal{X}_{1} & \equiv \frac{x_{1} \alpha\left(B^{\theta} B_{\theta}\right)_{1}}{2 B^{r} B_{r}\left(g_{t t}+g_{t \phi} \Omega_{F}\right)^{2}}, \quad \text { and } \\
\mathcal{T}_{1} & \equiv \frac{\sigma_{1}}{2}\left(\frac{u_{t}}{u^{r}}\right)_{1}^{2} \frac{\rho_{w}^{2}\left(\Omega_{1}-\Omega_{F}\right)}{g_{r r}\left(g_{t \phi}+g_{\phi \phi} \Omega_{F}\right)} .
\end{aligned}
$$

Here, we restrict ourselves to cold accretions $\left(P_{1}=0\right)$. Using the definition of $\zeta$ and the equation of state for a Boltzmann gas with polytropic index $\Gamma$,

$$
\begin{aligned}
& \mu_{1}=m_{\mathrm{p}} c^{2}, \quad \text { and } \\
& \mu_{2}=m_{\mathrm{p}} c^{2}\left(1+\frac{\Gamma}{\Gamma-1} \frac{P_{2}}{n_{2} m_{\mathrm{p}} c^{2}}\right),
\end{aligned}
$$


we find with Eq. (58)

$$
1-\frac{u_{2}^{t}}{u_{1}^{t}}=\sigma_{1}(q-1)-\frac{\Gamma}{\Gamma-1} \frac{g_{r r}\left(u^{r} / u^{t}\right)_{1}^{2}\left(u_{2}^{t}\right)^{2}}{\xi} \Pi
$$

Combining Eqs. (49) and (45) gives the quadratic equation for $\zeta$ $\zeta^{2}-\left\{1+\sigma_{1}+\left(\frac{x_{1}}{g_{t t}+g_{t \phi} \Omega_{F}}-\frac{\sigma_{1} \Omega_{F}}{\Omega_{F}-\Omega_{1}}\right) \xi\right\} \zeta+\left(\frac{x_{1}}{g_{t t}+g_{t \phi} \Omega_{F}}-\frac{\sigma_{1} \Omega_{1}}{\Omega_{F}-\Omega_{1}}\right) \xi=0$.

We are now able to eliminate $u_{2}^{t}$ and $q$ from Eq. (63). After considerable manipulations, we end up with a polynomial of seventh degree in $\xi$

$$
\sum_{i=0}^{7} c_{i}\left(u_{1}^{t}, \sigma_{1}, x_{1}, \Gamma ; m, a, \Omega_{F}\right) \xi^{i}=0
$$

The coefficients $c_{i}$ are dependent only on upstream parameters, except for $\Gamma$, which is a function of the downstream temperature (see Appl \& Camenzind 1988).

\section{Concluding Remarks}

We have discussed the general relativistic MHD shock conditions for accretion onto a rotating black hole. Here, we introduce the magnetization parameter seen by ZAMO, because the shock conditions are related to local plasmas and the magnetosphere is dragged by the rotating black hole. Then, we can obtain similar expressions as for the special relativistic case. However, our formulas include the Kerr metric in many places.

We have seen that the compression ratio $\xi$ is the solution of a polynomial of seventh degree. The polynomial (65) has, in general, several real solutions corresponding to the different shock transitions. Then, the downstream quantities $\zeta, q, u_{2}^{t}, u_{2}^{r}, \Omega_{2}$, and $\Pi$ are obtained from Equations, (64), (49), (52), (20), (50), and (58), respectively. The concrete expressions of $c_{i}$ and more detailed analysis will be presented by Rilett (in preparation).

In the future, we will discuss applications of the results for accreting plasmas in a black hole magnetosphere; that is, we will treat a multi-magnetosonic point solution with the shock formation.

\section{References}

Appl, S., \& Camenzind, M. 1988, A\&A, 206, 258

Bekenstein, J. D., \& Oron, E. 1978, Phys. Rev. D, 18, 1809

Blandford, R. D., \& Znajek, R. L. 1977, MNRAS, 179, 433

Camenzind, M., 1986, A\&A, 156, 137

Rilett, D., Takahashi, M., Fukumura, K., \& Tsuruta, S., in preparation

Takahashi, M., 2000, in Proc. 19th Texas Symp. on Relativistic Astrophysics and Cosmology, eds. E. Aubourg, T. Montmerle, \& J. Paul (Paris: World Scientific Press), in press 